\title{
Biocontrol opportunities to study microevolution in invasive
}

\section{populations}

\author{
Sze Huei Yek \& Bernard Slippers*
}

\begin{abstract}
Department of Genetics, Forestry and Agricultural Biotechnology Institute (FABI), University of Pretoria, Pretoria 0002, South Africa.

*Corresponding author: Bernard.Slippers@up.ac.za
\end{abstract}

Keywords: colonization success; experimental systems; biological control

Invasive populations have been actively used to test ecological and evolutionary hypotheses about colonization success in recent years. This is often complicated by the fact that many confounding factors, such as genetics, demographics and environmental variables influence the success of colonizing populations (see for example [1]). In a recent paper, Rius \& Darling [2] reflect on this challenge in the context of the role of admixture in invading populations and concludes that one of the largest gaps in the field is the lack of statistically robust experimentation in invading populations.

We argue that biological control systems offer an underexplored opportunity for experimentation with colonization success of insects and microbes. Factors determining colonization success are equally important in invasion biology and biological control systems. Apart from the fact that these systems share similar processes (Figure 1), both also have large ecological impacts and involve 
Biological control opportunities

- Collection and documentations of different parental populations (e.g. genotypes and environmental parameters)

- Development of rearing, monitoring and storing tools.

- Documentation of demographic and genetic effects of rearing (e.g. inbreeding, admixture)

- Details of life history traits

- Controlled experimentation with releases (e.g. different population sizes, number of releases)

- Reciprocal transplant/common garden experimentation (e.g. variable genotypes, environments)

- Documentation and monitoring of long-term consequences of genetic, demographic and life history traits

- Comparative studies across local and global distribution

\section{Colonization stages}

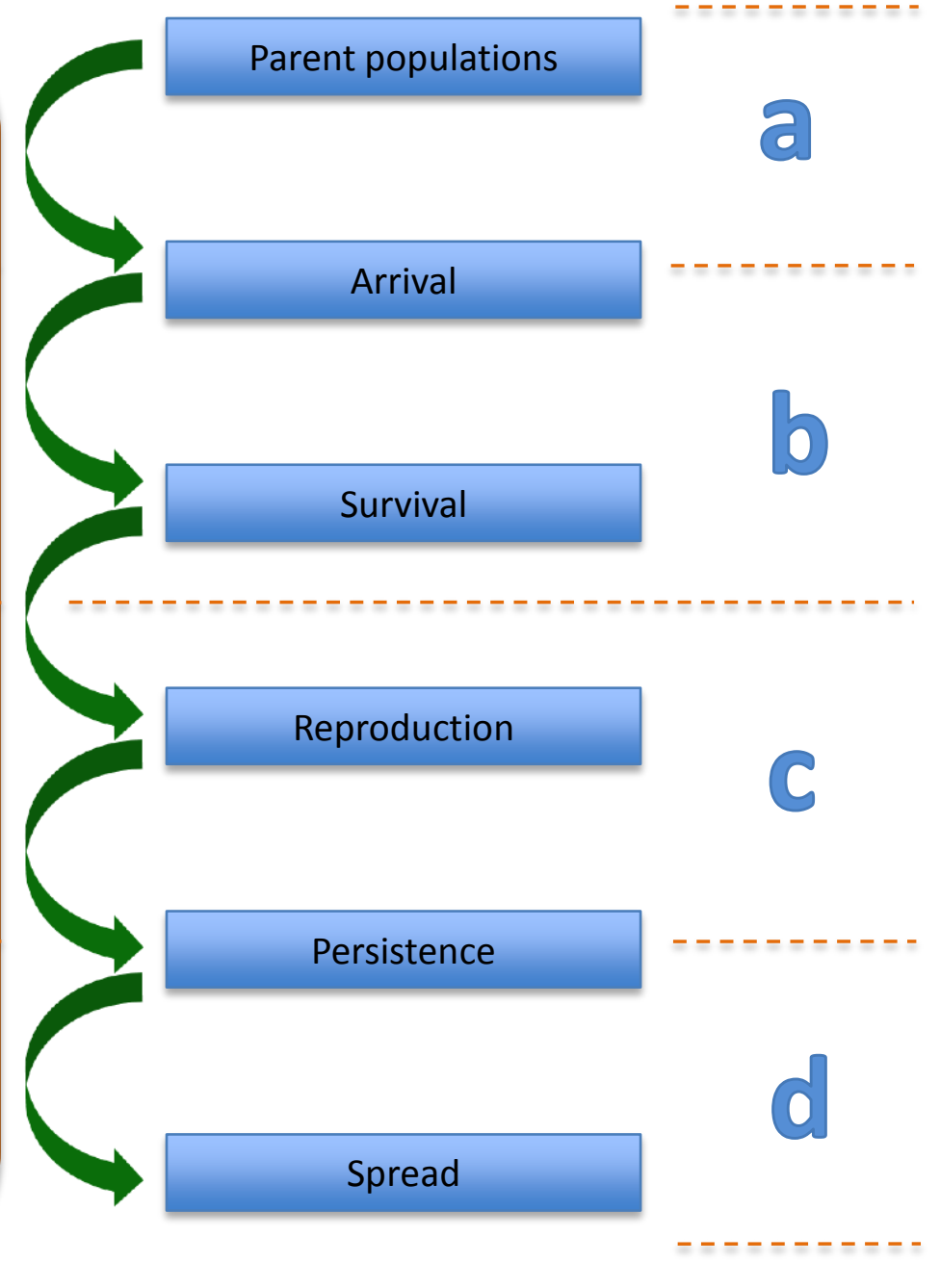

Figure 1

Opportunities offered by biological control to study microevolutionary processes during (a) collection, (b) rearing and quarantine studies, and (c) after release. These opportunities are related to the colonization stages by invasive populations referred to in Rius \& Darling [2]. The opportunities offered often overlap between colonization stages, for example development of tools in rearing, storing and monitoring would also offer opportunities during colonization stages after release. 
substantial financial investments. Biological control systems, however, allow for more precise documentation of relevant information all along the colonization processes. It also offers the opportunity to manipulate variables (e.g. genetics and demographics) that is often confounded in invading populations ([3] and papers discussed in the associated journal issue).

The colonization history of invasive species is required for defining and testing hypotheses concerning establishment successes. Due to incomplete historical data, the introduction routes are often reconstructed based on sparse observational events and inferred indirectly through molecular markers [4]. The stringent criteria for selection of biological control agents (e.g. [5]) ensure detailed documentations of the geographic locations of parental populations and their genotype lineages (Figure 1a). Through rearing (a required procedure for host specificity testing prior to release), populations consisting of different sizes and backgrounds can be generated to test factors influencing population establishment (e.g. [6]; Figure 1b, c).

To characterize the importance of demographic factors or genotypic variation for colonization success, for example, requires experiments in which different size populations and mixtures of levels of genetic variation are directly compared under the same environmental conditions, which is often impossible for practical, ethical or legal reasons in most invading populations (but see [1]). In biological control systems, field releases of populations with varying genotypes/sizes are feasible (Figure 1c), and even encouraged in some instances for predicting the efficacy of biological control agents (e.g. [7]). The ease of 
manipulation of biological control agents allow the design of experiments with replicated populations in controlled spatially heterogeneous environments, providing a valuable means to complement insights from invasive organisms.

The success of many invasive populations has often been attributed to multiple introductions and adaptation of novel genotypes (e.g. [8]), yet the data on longterm consequences of genetic diversity on these introduced populations are extremely rare. In contrast, biological control systems provide an excellent opportunity for large-scale evolutionary experiments and long-term field surveys (Figure 1c). Phenotypic changes, life-history trait variations, colonization succession and population expansion are just some of the data that can be gathered in a long-term biological control experimentations (for example see [9]).

The knowledge on life-history traits, tools for rearing, characterization and (in some cases) long-term storage of the organisms involved, growing genetic and genomic resources, and wide-scale (even global) application of some biological control populations represent excellent research capacity for many biological control systems (Figure 1; $[3,10]$ ). Given the overlap between invasion biology and biological control, using this opportunity is important both to understand and to manage the growing global threat from invasive insect pests and microbial pathogens $[11,12]$. 


\section{References}

1. Hufbauer, R.A. et al. (2013) Role of propagule pressure in colonization success: disentangling the relative importance of demographic, genetic and habitat effects. J. Evolution. Biol. 26, 1691-1699

2. Rius, M. and Darling, J.A. (2014) How important is intraspecific genetic admixture to the success of colonizing populations? Trends Ecol. Evol. 29, $1-10$

3. Roderick, C.K. et al. (2012) Evolution and biological control. Evol. Appl. 5, $419-423$

4. Estoup, A. and Guillemaud, T. (2010) Reconstructing routes of invasion using genetic data: why, how and so what? Mol. Ecol. 19, 4113-4130

5. Van Lenteren, J.C. et al. (2006) Assessing risks of releasing exotic biological control agents of arthropod pests. Ann. Rev. Entomol. 51, 609634

6. Grevstad, F.S. (1999) Experimental invasions using biological control introductions: the influence of release size on the chance of population establishment. Biol. Invasions 1, 313-323

7. McClay, A.S. and Balciunas, J.K. (2005) The role of pre-release efficacy assessment in selecting classical biological control agents for weeds applying the Anna Karenina principle. Biol. Control 35, 197-207

8. Dlugosch, K.M. and Parker, I.M. (2008) Founding events in species invasions: genetic variation, adaptive evolution, and the role of multiple introductions. Mol. Ecol. 17, 431-449

9. Phillips, C.B. et al. (2008) East meets west: adaptive evolution of an insect introduced for biological control. J. Appl. Ecol. 45, 948-956 
10. Roderick, G.K. and Navajas, M. (2003) Genes in new environments: genetics and evolution in biological control. Nat. Rev. Genet. 4, 889-899

11. Boyd, I.L. et al. (2013) The consequence of tree pests and diseases for ecosystem services. Science 342, 1235773

12. Fisher, M.C. et al. (2012) Emerging fungal threats to animal, plant and ecosystem health. Nature 484, 186-194 